\title{
Design of Automatic Lap Counting System for Track Events Based on Wireless Data Transmission
}

\author{
Zhang Zhan-Yi* \\ College of Physical Education of Henan Normal University, Xinxiang 453007, China
}

\begin{abstract}
The paper is to discuss the design content of automatic lap counting system for track events based on wireless data transmission. Starting from the current situation and existing problems of automatic lap counting system for track events, this paper discusses the common problems of automatic lap counting system and discusses how to establish automatic lap counting system for track events based on wireless data transmission from RF technique and wireless cluster network design. It is found from practice that, the automatic lap counting system established based on the integrated processing of the above two technologies can effectively meet the lap counting demands of all track events and has higher application value. It follows that, wireless data transmission technology has higher practical value in the design of automatic lap counting system for track events, and in-depth research should be made on it in future system design.
\end{abstract}

Keywords: Automatic lap counting system, RF technique, wireless cluster network design, wireless data transmission.

\section{CURRENT SITUATION AND EXISTING PROB- LEMS OF AUTOMATIC LAP COUNTING SYSTEM FOR TRACK EVENTS}

\subsection{Current Situation of Automatic Lap Counting Sys- tem For Track Events}

Many firms have started the research on automatic lap counting system for a long time and have put it into production practice. For instance, during Rotterdam Marathon, $135 \mathrm{kHz}$ passive RF technique was adopted, the wearable devices of athletes are very small in size and their production cost is very cheap, but such devices can only receive data effectively within $15 \mathrm{~m}$. Therefore, in Marathon, designers put card reader antenna array in a thin pat and pave on ground, and set transponders on the shoes of athletes, so as to be convenient for receiving signals $[1,2]$. But it is found that, since long-distance runners always wear spiked shoes, if athletes step on electric wire, communication devices will be damaged, and it is very possible to stumble athletes and influence competition results. A Chinese firm in Shenzhen effectively resolved equipment damage problem by paving antenna under the track. However, since such improvement will increase installation cost by a wide margin, it is difficult to be widely promoted.

\subsection{Existing Problems of Automatic Lap Counting Sys- tem for Track Events}

Further promoting automatic lap counting system in track events can effectively resolve the problems of existing manual lap counting [3]. However, the existing lap counting device only realizes a certain function of lap counter and cannot completely replace lap counter. In general, automatic lap counting system has the following problems: (1) high installation cost and a wide range of installation involved; (2) single system function cannot realize automation; (3) a lower degree of humanization design of operating interface and defective functional layout $[4,5]$.

\section{RF TECHNIQUE}

\subsection{Active RFID Technique}

\subsubsection{VE-FASI-A 433.92MHZ Active Device}

(1) VE-FASI-A 433.92MHZ active card reader

VE-FASI-A 433.92MHZ active card reader is mainly used to receive the signal sent by VE-FASI-A 433.92MHZ active tag and transfer the corresponding signal to exterior through special series port. Hence, VE-FASI-A 433.92MHZ active card reader constitutes an active identification and reading system jointly with active tag and host computer, and is widely applied in personnel management and resource management. It has the following features: (1) stable reading range, adjustable (reading range in open field $>30 \mathrm{~m}$, and that in door $\approx 10 \mathrm{~m}$ ) ; (2) strong anti-collision capacity, with maximum capacity of $125 / \mathrm{s}$; (3) small in size, easy for taking.

\section{(2) Directional antenna A520}

Directional antenna A520 is designed by linear vertical polarization technique. When matching up with card reader, it realizes directional active tag reading in a certain range. Currently, the reading range of directional antenna A520 is: level $85^{\circ}$, vertical $120^{\circ}$, front-to-rear ratio $>15 \mathrm{~dB}$, and it can pass through non-metallic objects to receive data.

\section{(3) Active tag}

VE-TAI-1 433.92MHZ active tag is used to send its ID information periodically to VE-FASI-A 433.92MHZ active 
reader, and it constitutes active identification and reading system jointly with active reader and the host computer.

\subsubsection{Application of Anti-Collision Principle in Automatic Lap Counting System}

To ensure the card reader can identify tag effectively, multiple ways should be adopted to avoid collision and lay a foundation for the transmission of data between transponder and reader. Combined with ALOHA algorithm, this paper analyzes the application of anti-collision principle.

The multi-access mode adopted by ALOHA algorithm is an irregular multi-access processing, applies to readers. Only one data package is available, the data package will be sent to the reader from the transponder. Such data transmission process exists for a long time, and data transmission is done continuously in a cycle period, so as to prevent from collision of data package during transmission.

Generally, the quantity of exchange data packages of ALOHA algorithm is consistent with that of transponders sent as per the formula within required time. G, average content of data package is consistent with the average value of $\mathrm{T}$, special time, and the calculation formula of $\mathrm{G}$ is:

$\mathbf{G}=\sum_{1}^{\mathrm{n}} \frac{\tau_{\mathrm{n}}}{\mathrm{T}} \mathbf{r}_{\mathrm{n}}$

In Formula (1), $\tau$ refers to the transmission time of data package; $n$ refers to the quantity of transponders $(1,2,3,4$ ...); ${ }^{\mathrm{r}} \mathrm{n}$ refers to the quantity of data package sent to transponder within the set time $\mathrm{T}$.

RFID reader is used for CPU chip to control wireless receiving circuit, conduct real-time monitoring on network, and transmit data through special port. See Fig. (1) for its schematic diagram.

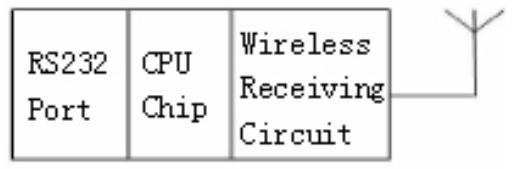

Fig. (1). Schematic diagram of card reader.

Based on Fig. (1), tag CPU control wireless transmitter circuit will send its ID information to outside periodically. See Fig. (2) for its schematic diagram.

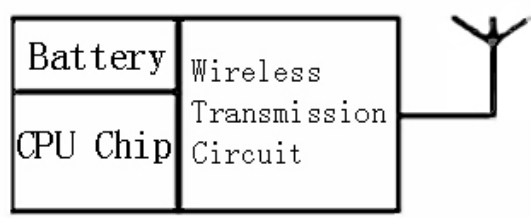

Fig. (2). Schematic diagram of tag.

When athletes are running, the system will record the running action of each athlete. Currently, the radius of curve of standard $400 \mathrm{~m}$ track is $36.5 \mathrm{~m}$ and a safety zone of 1 to $1.2 \mathrm{~m}$ should be set in the inner side of the track. Therefore, the antenna here is set in the inner side and $1.5 \mathrm{~m}$ from the track. Combined with directional antenna, the tag within level $85^{\circ}$ and vertical $120^{\circ}$ will be read. To ensure enough reading time of the system, the side in horizontal range of the antenna should coincide with the end point, so as to ensure effective reading range of $120^{\circ}$. Since the effective receiving range of the antenna in the study is $30 \mathrm{~m}$, the effective receiving range of the system is the length from Point A to the end point. See Fig. (3).

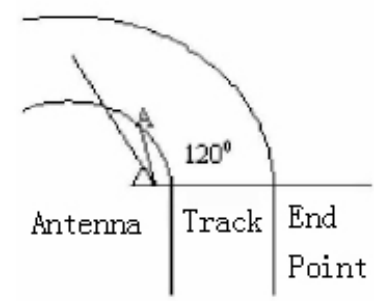

Fig. (3). Schematic diagram of system receiving range.

\subsection{Design of Card Reader Data Receiving and Process- ing Software}

According to the contents of automatic lap counting system for track events, this paper sorts out the basic design flow of card reader data receiving and processing software. See Fig. (4) for details.

\section{DESIGN OF WIRELESS CLUSTER NETWORK}

Currently, most Chinese computer local area network communications adopt wire communication. Wire communication has the advantages of high security and reliability of data transmission. But wire communication requires laying more open wires. Limited by natural condition and environmental factor, the wiring is difficult for some areas, but wireless communication can effectively resolve the problem.

\subsection{Wireless Data Transmission Module}

\subsubsection{Wireless Module Pin Definition}

FC-201/SA provides one 10-pin connector (connecting terminal), it can be defined as:

Pin 1: GRD to terminal GND; Pin 2: serial data receiving terminal to system terminal; Pin 3: data transmission terminal to terminal; Pin 4: CTS directly to terminal; Pin 5: onsite test of strong signal field received by RSSI; Pin 6: SQ squelch indication; Pin 7: RxD of RS232 directly to terminal; Pin 8: TxD of RS232 directly to terminal; Pin 9: power VCC; Pin 10: signal reset (GND).

In the entire system, wireless data transmission module is connected to the host computer through TEL interface, and serial data receiving terminal is connected to the control chip.

\subsubsection{Application of FC-201/SA}

FC-201/SA communication channel is featured by halfduplex. It applies to multilink point-to-point communication processing. When applying such technique, one master station and some slave stations should be set successively. All slave stations should have the same address.

Communication coordination should be controlled completely by wireless station. Commands should be sent with 


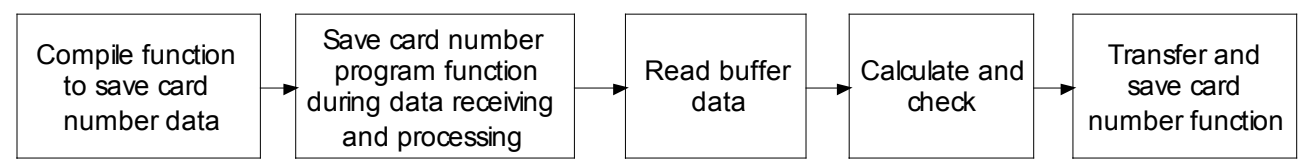

Fig. (4). Design flow of card reader data receiving and processing software.

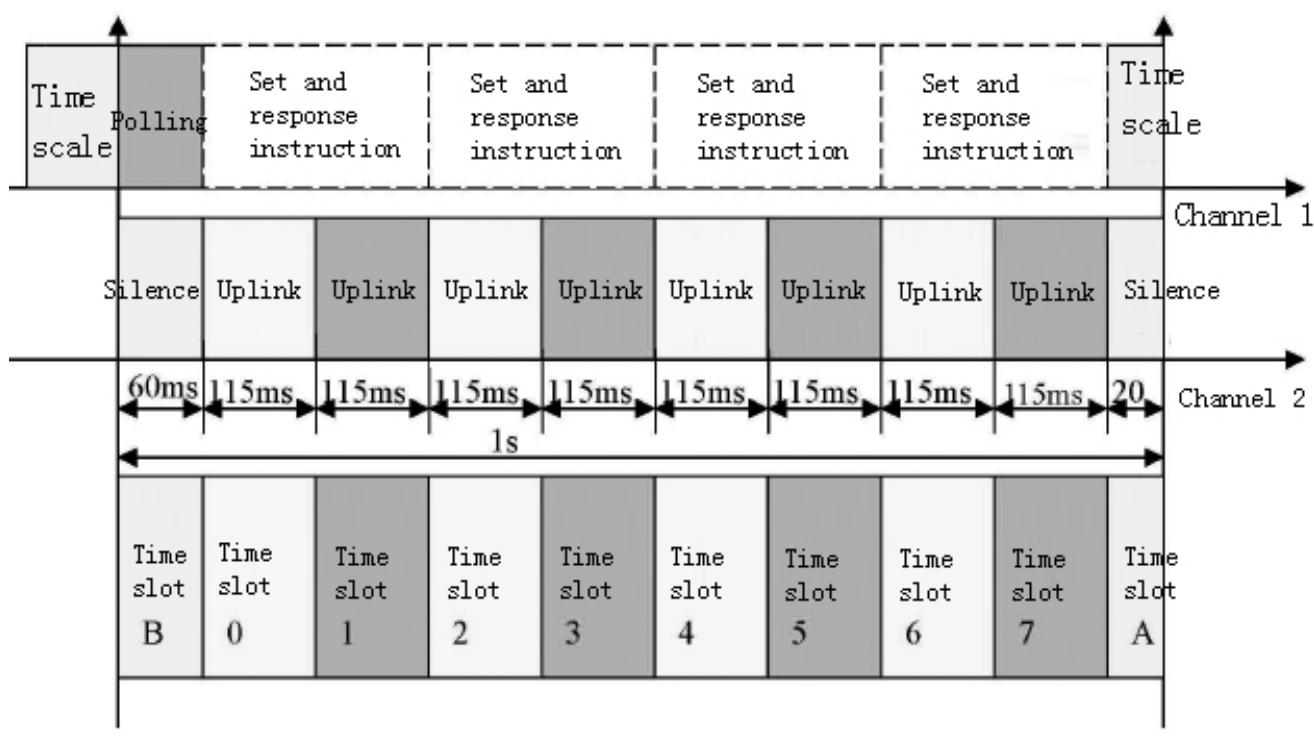

Fig. (5). Division of wireless communication channels in normal condition.

the data attached with address code. After being received completely by the host computer, compare all address codes with local address codes and process the data with inconsistent address codes (the system will not respond); same address codes signifies local data, and the system will respond the data according to the established procedure. The process should be completed as per upper layer protocol. And it should ensure that, in a certain moment, only one radio in the communication network is at the transmitting state, so as to avoid mutual interference of radios.

\subsection{Wireless Cluster Network Communication Protocol}

Communication protocol is an agreement concluded by both communication parties, including synchronization mode, data transmission speed, error collecting mode, etc., and both parties should complete the designated operation in a unified standard. Currently, most Chinese computer local area network communications mainly adopt wire communication. Wire communication has the major advantage of high reliability of data transmission. But in actual application, more open wires should be erected. But it is difficult to erect open wires in certain special fields (such as lap counting for track events), then wireless communication, especially wireless network communication protocol, can be adopted to resolve the problem.

\subsubsection{Working Mode of Wireless Communication System}

Aiming at the design specifications and requirements of the system, combining with the features and actual situation of automatic lap counting system, the paper thinks "FDMA" and "TDMA" should be combined for managment to improve the working mode of wireless communication system.

(1) Time slot of wireless communication
When downlink communication chain sends synchronization information periodically, wireless terminal makes improvements as per the feature of the information, is divided into multiple time slots within the same period, which are allocated to different functional modules for use. This paper summarizes the general division of communication time slots. See Fig. (5).

Since there is certain power establishment time in the process of wireless device receiving and sending instructions, the division of channels during the process must also be considered. When downlink communication chain sends time scale information, wireless terminal will make proper changes according to the time scale information. $1 \mathrm{~s}$ is divided into 10 time slots, and $A B$ time slot is $80 \mathrm{~ms}$, which can be used for sending time slot information.

"TDMA" can further save channel resources, but it is also easy to be interfered [6]. Hence, to ensure the reliability of system running, "FDMA" is introduced based on it, and its advantage of multi-frequency clock is utilized to prevent channels from interference. During information exchange, "Polling" and competitive mix should be adopted to make real-time data exchange. From the prospective of signaling, wireless network signals can be divided into two parts: signaling control channel and data exchange channel. The entire communication chain can be divided into two channels. Channel 1 is mainly responsible for signaling control, namely, downlink channel and Channel 2, uplink channel, is mainly responsible for data retransmission.

\section{(2) Definition of data frame structure}

In the protocol, the basic structure of data frame should be defined to provide a foundation for data transmission. This paper summarizes partial contents of the definition of data frame structure. See Table 1 for details. 
Table 1. Definition of data frame structure.

\begin{tabular}{|c|c|c|}
\hline SN & Structure & Basic Contents \\
\hline \hline 1 & Frame header & 2 bytes, $0 * 50$ and $0 *$ BC separately \\
\hline 2 & Frame size & 1 byte, actual size of the frame \\
\hline 3 & Frame technique & 2 bytes, be able to send count value instructions \\
\hline 4 & Originator ID & $\begin{array}{c}1 \text { byte, contents are expressed by four bytes in high order, including host com- } \\
\text { puter, card reader, etc. }\end{array}$ \\
\hline
\end{tabular}

\subsubsection{Instructions of Wireless Cluster Network Communi- cation System}

Wireless cluster network instructing is a major part of the instructions of network communication system. It sends instructions through the wireless terminal of communication center, including time scale instructions, response instructions, etc.

(1) Time scale instruction: control word: $0 * \mathrm{~A}$; information word: 1 byte, software version number, 2 bytes: the unit is second, the center computer starts time increment after starting counting, and the system will save and reset time automatically according to requirements when the competition restarts. (2) Response instruction: control word: $0 * \mathrm{C}$; information word: 1 byte, terminal ID; 1 byte, number of response frames.

Through communication protocol, complex software framework can be completed on the relevant chain of communication system, therefore, the normal work of any communication system can not be without the support of communication protocol. Wireless module test shows that, the relevant design in this paper is correct and reliable, and can realize information receiving and sending among wireless modules.

\section{CONCLUSION}

This paper discusses automatic lap counting system for track events based on wireless data transmission technique and analyzes automatic lap counting system for track events from RF technique and wireless cluster network in detail.
Designers should start from financial demand and technical supply, and comprehensively consider the design requirements of multiple systems, so as to ensure scientificity and effectiveness of system design.

\section{CONFLICT OF INTEREST}

The author confirms that this article content has no conflict of interest.

\section{ACKNOWLEDGEMENTS}

Declared none.

\section{REFERENCES}

[1] X. Liu, and Kun Yang, "Design of Automatic Timing System for Track Events Based on End Point Spurt Image," Mathematical Techniques and Application (Academic Forum), no. 12, 2013.

[2] T. Wang, "Design of New Timing System for Track Events," Bulletin of Sport Science \& Technology, vol. 22, no. 03, 2014.

[3] Wang Qiufen \& Yuan Dongfeng. "Study on Automatic Scheduling Algorithm of Track Events in Colleges and Universities," Computer and Modernization, no. 02, 2012.

[4] R. Gao, L. Yin, and J. Zhang, "Design and Realization of A Dynamic Grouping Algorithm Based on Track Events," Software, vol. 31, no. 01, 2011.

[5] Y. Fu, and F. Li, "Analysis on the Application of Photofinish in Modern Track and Field Athletics," New Technologies and Products, no. 07, 2010.

[6] M. Li, Xiong Wei, Liang Qing et al. "Study on Wireless Sensor Network DV-Hop Location Algorithm," Journal of Air Force Engineering University (Natural Science Edition), vol. 08, no. 04, 2012. 